\title{
Measurements of fission fragment properties using RPl's lead slowing down spectrometer
}

\author{
C. Romano ${ }^{1}$, Y. Danon ${ }^{1, a}$, R. Block ${ }^{1}$, E. Blain ${ }^{1}$, and E. Bond ${ }^{2}$ \\ 1 Gaerttner LINAC Laboratory, Rennselaer Polytechnic Institute, Troy, NY 12180, USA \\ 2 Los Alamos National Lab., Los Alamos, NM 87545, USA
}

\begin{abstract}
A double sided Frisch-gridded fission chamber for use in RPI's lead slowing-down neutron spectrometer (LSDS) is being developed at Rennselaer Polytechnic Institute. Placing this fission chamber in the high neutron flux of the LSDS allows the measurement of the energy dependent, neutron induced fission cross sections, as well as the mass and kinetic energy of the fission fragments of various small samples. The fission chamber consists of two anodes shielded by Frisch grids on either side of a single cathode. The sample is located in the center of the cathode and is made by depositing small amounts of actinides dissolved in solution on very thin films. The chamber was successfully tested and calibrated using $0.4 \mathrm{ng}$ of ${ }^{252} \mathrm{Cf}$ and mass distributions were compared to previous work. As a proof of concept, the chamber was then placed in the LSDS to measure, simultaneously, the neutron induced fission cross section and fragment mass and energy distributions of $24.9 \mu \mathrm{g}$ of ${ }^{235} \mathrm{U}$. The mass distribution as a function of neutron energy was examined and it may be possible to see changes in the mass distribution as a function of neutron energy. This process will enable the measurement of isotopes that are not available in large enough quantities (sub-micrograms) or with small fission cross sections (microbarns).
\end{abstract}

\section{Introduction}

The double Frisch-gridded fission chamber has been used successfully in the past to measure mass and energy of fission fragments [1-4]. However, the high flux of the LSDS at RPI [5] provides the additional benefit of being able to measure small samples, or those with small cross sections. The additional benefit is the ability to measure the neutron energy dependent cross section simultaneously with the fission fragment energies. This will enable the detailed comparison of mass spectra changes as a function of incident neutron energy, particularly in the resonance regions.

The chamber was first tested with ${ }^{252} \mathrm{Cf}$. This verified the function of the chamber and the development of the data analysis procedure. The tests with the ${ }^{235} \mathrm{U}$ sample in the LSDS verified that the concept of simultaneous measurement of fragment mass and cross section are feasible. Once the data analysis of the ${ }^{235} \mathrm{U}$ sample is complete, then measurements of actinides not previously measured can be performed.

\section{Fission chamber and electronics}

The fission chamber consists of two anodes shielded by Frisch grids on either side of a single cathode and filled with $\mathrm{CH}_{4}$. The sample is deposited on a $2500 \AA$ thick polyimide film located in the center of the cathode so that the fragments are emitted into each side of the chamber. The distance from the cathode to the grid is $27 \mathrm{~mm}$ which ensures that the fission fragments deposit their total energy in the gas. The grid signal is a function of the angle of emission and the anode signal is proportional to the energy of the fragment. The grid signal

a Y. Danon, e-mail: danony@rpi .edu is bipolar, so the sum of the anode and grid signal is used to process the data.

The signals from the chamber are amplified with Cremat 110 preamplifiers [6] that have been modified to a shaping constant of $500 \mathrm{nsec}$, which is the maximum time needed to collect the entire energy deposited in the gas. The cathode signal provides a gate to the ADC as well as a trigger to the event scaler which records events as a function of the neutron slowing time. The fragment energies and neutron slowing down time are recorded for each pulse by RPI's data acquisition system. The files are then prepared for offline analysis.

\section{$3^{252} \mathrm{Cf}$ measurements and data analysis}

Testing of the chamber and data acquisition system has been accomplished with a ${ }^{252} \mathrm{Cf}$ sample. The anode and sum pulse height distributions for ${ }^{252} \mathrm{Cf}$ are shown in figure 1 for the bare sample side of the chamber. The anode signal is subtracted from the sum signal to obtain the grid signal, and the angle is extracted from this through a procedure discussed in refs. [1-3]. The resulting angular distribution is shown in figure 2 and this data is used to correct for the energy lost in the sample as a function of the angle of emission.

The actual fragment energy can be determined using the following equation

$$
E=k A+\frac{\Delta E}{\cos (\theta)}+P H D(m, E)
$$

where $A$ is the anode pulse height, $k$ is the calibration factor from channel to energy, $\Delta E$ is the average energy lost in the sample or sample plus film when the fragment is emitted vertically, $\cos (\theta)$ is the fragment angle of emission and the $P H D$ is the pulse height defect. The pulse height defect is 


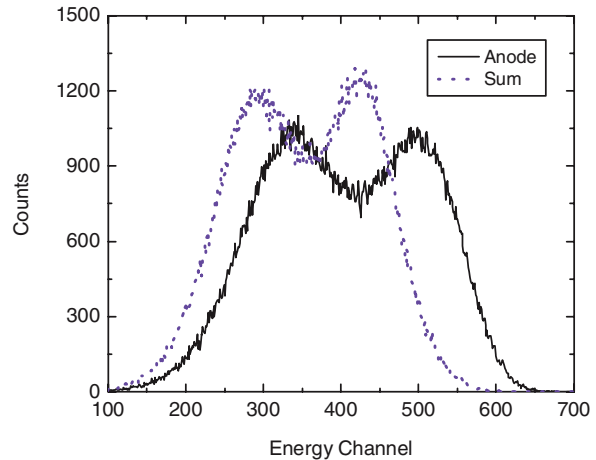

Fig. 1. Uncorrected anode and sum (anode + grid) pulse height distributions for the bare side of the ${ }^{252} \mathrm{Cf}$ sample.

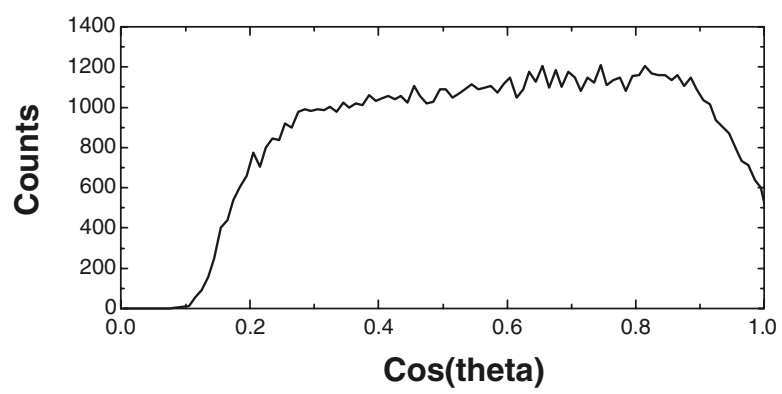

Fig. 2. Angular distribution of fission fragments of the ${ }^{252} \mathrm{Cf}$ sample.

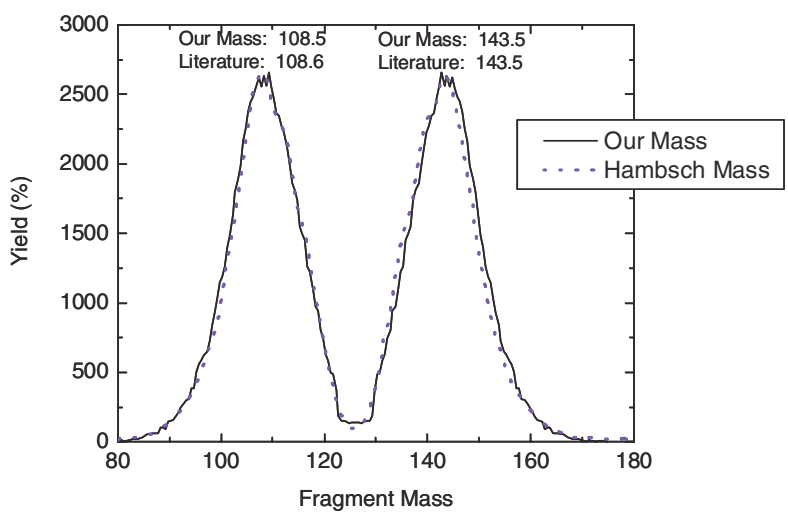

Fig. 3. Comparison of mass distributions of ${ }^{252} \mathrm{Cf}$ spontaneous fission to previous data.

a characteristic of the fill gas and chamber dimensions as well as fragment mass and energy. The pre-neutron emission mass is then determined from an iterative procedure beginning with the ratios of the two post neutron emission energies and average neutron emission data as a function of fragment mass. The resulting mass distribution for ${ }^{252} \mathrm{Cf}$ is shown in figure 3 and is compared to previous data from ref. [4]. The excellent correlation of the mass distributions with previous work indicates that the chamber and electronics are working properly and the data analysis is accurate.

\section{Sample size and preparation}

The benefit of the LSDS is that the high neutron flux allows measurement of very small samples. Recently at LANL, the neutron induced fission cross section of $10 \mathrm{ng}$ of ${ }^{239} \mathrm{Pu}$ was measured [7]. The uniqueness of this experiment at RPI is that the gridded fission chamber placed in the LSDS is able to measure both the neutron induced cross section and the mass and energy of the fission fragments at the same time. This can also be done with small samples.

The samples are made by dissolving small amounts of actinide in a solution, dropping it on a $2500 \AA$ polyimide film, and allowing it to evaporate. The ${ }^{252} \mathrm{Cf}$ sample was made at RPI by dissolving $4 \mathrm{ng}$ of ${ }^{252} \mathrm{Cf}$ in $0.1 \mathrm{M} \mathrm{HCl}$ and depositing $0.41 \mathrm{ng}$ on the film. The ${ }^{235} \mathrm{U}$ sample was made at LANL by dissolving $99.89 \%{ }^{235} \mathrm{U}$ in absolute ethanol. The resulting sample is $24.9 \mu \mathrm{g}$ over an area of approximately $1.5 \mathrm{~cm}^{2}$. Both sample sizes were determined through gross alpha counts.

\section{Experiments in the LSDS}

For the ${ }^{235} \mathrm{U}$ measurements, the fission chamber is placed in the LSDS which is a 75 ton, $1.8 \mathrm{~m}$ cube of lead. The lead is covered with a thin layer of Cadmium to prevent thermalized neutrons from reentering the lead. The RPI $60 \mathrm{MeV}$ Linac creates neutrons through a $(\gamma, \mathrm{n})$ reaction when the electrons interact with a tantalum target inside the lead spectrometer. The neutrons then slow down by scattering in the lead creating a large isotropic flux due to the fact that a neutron can pass through the sample many times. The resulting neutron flux is about 4 orders of magnitude larger than an equivalent $(5.6 \mathrm{~m})$ time-of-flight experiment. The high neutron flux allows for the measurement of isotopes that are not available in large quantities (submicrograms) or with small fission cross sections (microbarns).

LANL has recently obtained a LSDS that is a $1.2 \mathrm{~m}$ cube of lead. Work is being done there on neutron induced fission as well as $(\mathrm{n}, \alpha)$ cross section measurements $[8,9]$.

\subsection{Challenges of working in the LSDS}

The LSDS is a difficult working environment due to the large amount of brehmstrahlung radiation (gamma flash) created when the electrons impact the tantalum target as well as the high neutron flux. Gaseous detectors are resilient in this environment; however, the larger the volume of gas, the longer the recovery time of the system. The volume of gas is highly ionized by the gamma flash which causes the preamps to be saturated and then oscillate. However, a fast recovery time is required to take measurements at high neutron energies. Recovery time has been minimized in several ways. First, $\mathrm{CH}_{4}$ is used as the fill gas due to its high electron drift velocity, second a preamp with the fastest shaping constant that will allow the integration of the entire charge for each pulse is used. In our case, this is $500 \mathrm{~ns}$. A fast, pulsed switch is also placed between the chamber and the preamp to ground the signal during the gamma flash. This is not a perfect solution because the switch also creates a large pulse when turning on, but it 


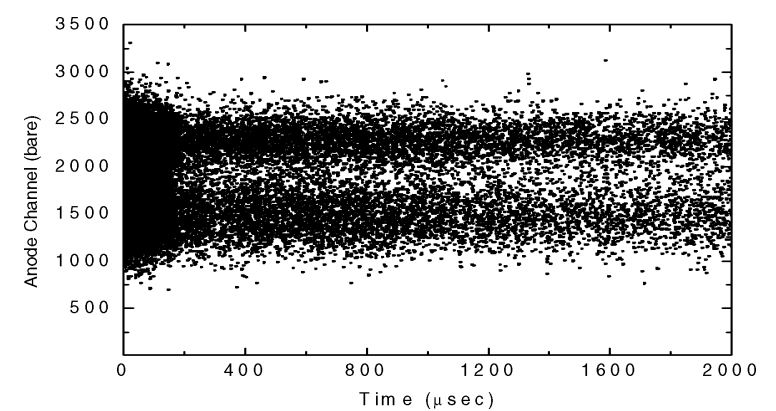

Fig. 4. Anode energy distribution as a function of neutron slowing down time in the LSDS.

does improve recovery time by about $20 \mu \mathrm{s}$. Our recovery time is approximately $12 \mu \mathrm{s}$ for energy measurements, and $5 \mu \mathrm{s}$ for cross section measurements.

\section{$6{ }^{235} \mathrm{U}$ measurements}

Using the calibrated gridded chamber, measurements of ${ }^{235} \mathrm{U}$ were made in the LSDS. The Linac was run at $8 \mu \mathrm{A}$ delivering $58 \mathrm{MeV}$ electrons at $180 \mathrm{~Hz}$. Simultaneous cross section and energy measurements were obtained in a single 4 hour run. Figure 4 shows a scatter plot of the fragment energy measured by the bare sample side anode verses neutron time of flight. The two bands are the energy of the light and heavy fragment and the decrease in the number of events as a function of slowing down time can be seen. The large number of data points early in the slowing down time is just due to the high neutron flux at that time.

\subsection{Cross section}

The neutron energy as a function of slowing down time of the neutron flux in the LSDS [5] is determined by the equation

$$
E(t)=\frac{k}{(t+0.3)^{2}}
$$

where $k=165000 \mathrm{keV}-\mu \mathrm{s}^{2}$ and $t$ is in units of $\mu \mathrm{s}$ and $E$ is in units of $\mathrm{keV}$. The energy dependent flux [5] is given as

$$
\phi(E)=k E^{-0.776} e^{-\sqrt{0.283} / E} d E
$$

where $k$ is the same as above. These equations are then used to determine the energy dependent neutron induced fission cross section as a function of neutron slowing down time. Measurements for this experiment can be obtained for neutron energies between $0.1 \mathrm{eV}$ and $10 \mathrm{keV}$. The neutron resolution (FWHM) in this energy range is approximately $35 \%$. This type of measurement was successfully accomplished in the past for ${ }^{247} \mathrm{Cm},{ }^{254} \mathrm{Es}$, and ${ }^{250} \mathrm{Cf}[10]$.

The preliminary cross section data for ${ }^{235} \mathrm{U}$ in the gridded chamber is shown in figure 5 compared to a previous cross section measurement in the LSDS. The results are in good agreement with previous [11] data measured at RPI.

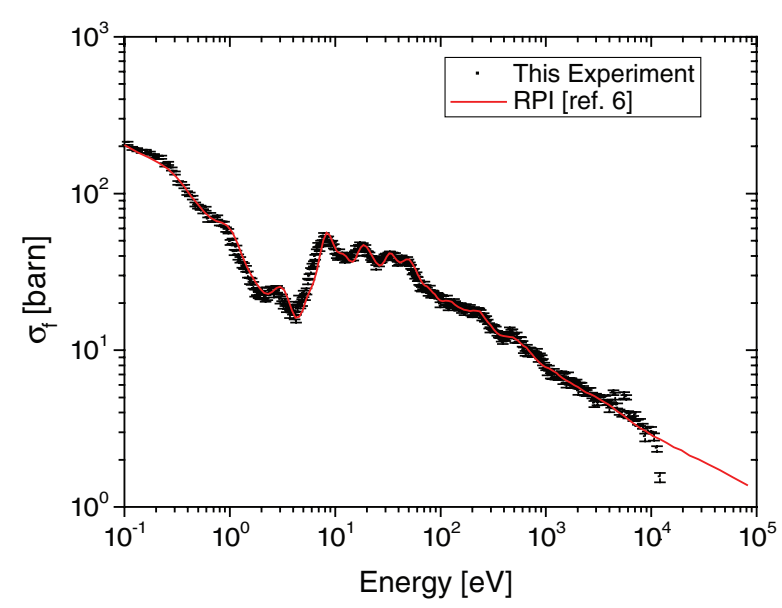

Fig. 5. ${ }^{235} \mathrm{U}$ cross section compared to previous cross section measurement in the LSDS at RPI.

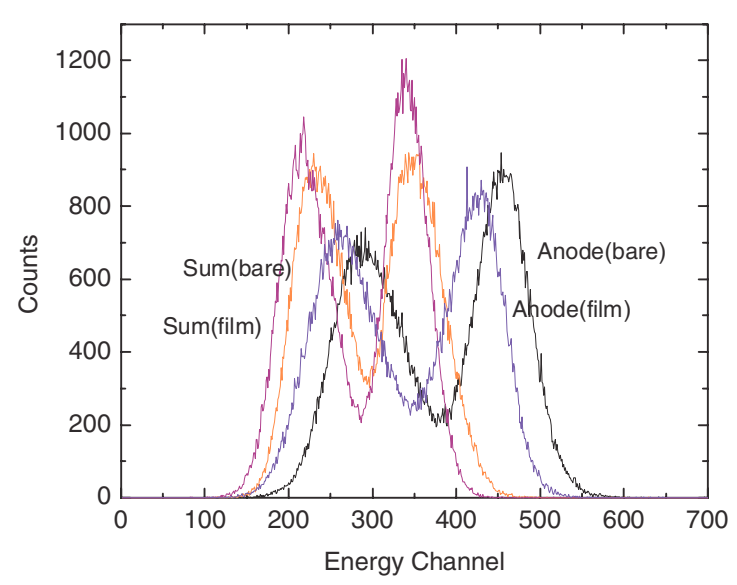

Fig. 6. Uncorrected anode and sum spectra for ${ }^{235} \mathrm{U}$.

\subsection{Fission fragment energy data}

Figure 6 shows the anode and grid pulse height distributions for both sides of the chamber summed over all slowing down times. The shift in energy between the two anode distributions is due to the fact that additional energy is lost by the fragments that must travel through the sample film. Extraneous pulses caused by backscatter in the sample or fragment emission at very high angles in the chamber have been filtered from this data by requiring the value of sum/anode to be within a range of 0.6 to 1 for each event.

The fission fragment energies as a function of $\cos (\theta)$, where $\theta$ is measured from normal to the sample, is shown in figure 7 . The separation of the heavy and light fragments is very apparent. The light fragment extends from about $\cos (\theta)$ of 0.2 to 1 . This is preliminary data and it can be seen that the derivation of the emission angle has not yet been perfected due to the fact that the heavy fragment data does not extend through the same range of $\cos (\theta)$. This calculation was accomplished for the ${ }^{252} \mathrm{Cf}$ measurement; however, the ${ }^{235} \mathrm{U}$ sample is an order of $10^{4}$ larger than the ${ }^{252} \mathrm{Cf}$ sample. Therefore, the energy of the fragment is much more sensitive to the angle of emission. 


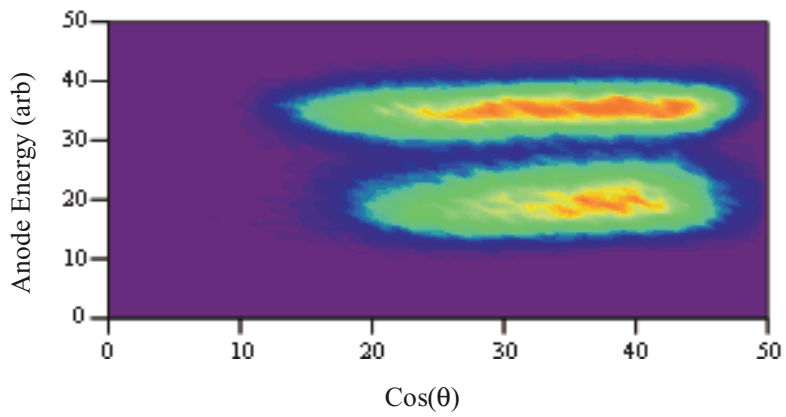

Fig. 7. Fission fragment energy as a function of $\cos (\theta)$. The range of the $\mathrm{x}$ axis is from $\cos (\theta)=0$ to $\cos (\theta)=1$ at $\mathrm{x}=50$.

\subsection{Mass distributions}

Preliminary calculations have been made for the fragment post prompt neutron emission mass distributions. Once the angular dependent energy is determined precisely, the mass distributions can be calculated.

\section{Summary}

These experiments show that the double-gridded fission chamber is capable of working in the LSDS environment and measuring simultaneously the fragment energy and mass distributions as well as cross section as a function of neutron slowing down time. This can be a valuable tool to measure very small samples in the unique LSDS environment. Future intended measurements include ${ }^{239} \mathrm{Pu}$.
We would like to acknowledge the technical staff at the RPI Gaertner Linac Laboratory, Peter Brand, Matthew Gray, and Martin Strock for the dedicated operation of the Linac during these experiments.

\section{References}

1. C. Budtz-Jorgensen, H. Knitter, NIM A 223, 295 (1984).

2. C. Budtz-Jorgensen, H. Knitter, C. Streade, F. Hambsch, R. Vogt, NIM A 258, 209 (1987).

3. F. Tovesson, F.J. Hambsch, S. Oberstedt, U. Bax, J. Nucl. Sci. Technol. Suppl. 2, 673 (August 2002).

4. F.J. Hambsch, J. Van Aarle, R. Vogt, NIM A 361, 257 (1995).

5. R.E. Slovacek, D. Cramer, E. Bean, R. Hockenbury, J. Valentine, R. Block, Nucl. Sci. Eng. 62, 455 (1977).

6. http://www.cremat.com/.

7. D. Rochman, R.C. Haight, J.M. O'donnell, S.A. Wender, D.J. Vieira, E.M. Bond, T.A. Bredeweg, J.B. Wilhelmy, T. Granier, T. Ethvignot, M. Petit, Y. Danon, C. Romano, NIM A 564, Issue 1, 400 (2006).

8. Y. Danon, C. Romano, J. Thompson, T. Watson, R.C. Haight, S.A. Wender, D.J. Vieira, E. Bond, J.B. Wilhelmy, J.M. O'Donnell, A. Michaudon. T.A. Bredeweg, T. Schurman, D. Rochman, T. Granier, T. Ethvignot, J. Taieb, J.A. Becker, NIM B (2007) (to be published).

9. C. Romano, Y. Danon, R.C. Haight, S. Wender, D. Vieira, E. Bond, R. Rundberg, J. Wilhelmy, J. O'Donnell, A. Michaudon, T. Bredeweg, D. Rochman, T. Granier, T. Ethvignot, NIM A 562, Issue 2, 771 (2006).

10. Y. Danon, R.E. Slovacek, R.C. Block, R.W. Lougheed, R.W. Hoff, M.S. Moore, Nucl. Sci. Eng. 109, 325 (1991).

11. Y. Danon, Master's Thesis, Rensselaer Polytechnic Institute (1990). 